\title{
SOME CONTRIBUTION TO STUDY OF GENERAL RELATIVE PRIME AND WEAKLY PRIME BI-HYPERIDEALS IN ORDERED HYPERSEMIGROUPS
}

\begin{abstract}
A. BASAR ${ }^{1, *}$, M. Y. ABBASI ${ }^{2}$, B. SATYANARAYANA ${ }^{3}$, P. K. SHARMA ${ }^{4}$, S. A. KHAN ${ }^{2}$
AbStRaCt. In this paper, we introduce $G$-hyperideal, $G$-prime hyperideal, $G$-prime-bihyperideal, and $G$-weakly prime hyperideal in ordered semihypergroups and we also define $G$-regular ordered hypersemigroups as well as $G$-semisimple ordered hypersemigroups. Thereby, we extend the results to the broader and abstract setting of ordered hypersemigroups, this also strengthens previous generalizations relating to rings, semigroups and other algebraic structures.
\end{abstract}

\section{INTRODUCTION}

Dedekind [21], [22], [26] pioneered and invented the related notions of ideal and prime ideal by deriving its motivation and name from kummer's ideal numbers. They were a generalization of the concept of ideal numbers as a special subgroup of a ring defined by Kummer [21]. Later, this concept was expanded by Hilbert and especially, by master algebraist Noether: the mother of modern algebra [24]. Noether [18], in her seminal paper, defined and developed the theory of ideals in commutative rings. Noether and Artin gave modern definition of such fundamental notions as one sided ideal and ideal along with module and chain condition [23]. McCoy [41] studied prime ideals in general rings. The concept of a bi-ideal on semigroup was introduced by Good and Hughes [49]. The notion of bi-ideal and generalized bi-ideal in semigroups was introduced and studied by Lajos [50], [51]. Thereafter, the concept of a quasi-ideal was introduced by Steinfeld [42], [43] on rings and semigroups.

The notion of ordered semigroups was introduced and studied by Conard [46]. The notion of an ordered quasi-ideal in ordered semigroups was introduced by Kehayopulu et al. in their papers [33], [37]. They studied theory of ordered semigroups based on ordered ideals analogous to the theory of semigroups based on ideals. For regular ordered semigroups, left regular

\footnotetext{
${ }^{1}$ Department of Natural and Applied Sciences, Glocal University, Saharanpur, UP, India

${ }^{2}$ Department of Mathematics, Jamia Millia Islamia, New Delhi, India

${ }^{3}$ Department of Mathematics, Acharya Nagarjuna University, Guntur, A.P., India

${ }^{4}$ PG Department of Mathematics, D. A. V. College, Jalandhar, Punjab, India

${ }^{*}$ Corresponding AUthor

E-mail address: basar.jmi@gmail.com, mabbasi@jmi.ac.in, bhavanari2002@yahoo.co.in,

pksharma@davjalandhar.com, khansabahat361@gmail.com.

Key words and phrases. semigroup, hypersemigroup; hyperideal, $G$-hyperideal; weakly prime ideal; $G$-weakly prime hyperideal; prime-bi-hyperideal; semiprime bi-hyperideal; relative prime $G$-hyperideal.
}

Received 21/12/2021. 
ordered semigroups and intra-regular ordered semigroups, we refer [17], [32], [34], [35], [36], [37].

Hyperstructure theory was introduced by Marty [19]. Algebraic hyperstructures are a natural generalization of classical algebraic structures. Hyperstructure has wide ranging applications in mathematics as well as in other disciplines that is introduced and discussed in the reference book by Corsini and Leoreanu [44]. In a classical algebraic structure, the composition of two elements is an element, while in an algebraic hyperstructure, the composition of two elements is a set. Semihypergroups is one of the most active fields of research in the area of hyperalgebraic structures. A recent monograph by Davvaz [14] introduced basic ideas and techniques required to study hypersemigroups and is a suitable reference book for researchers and scholars. Semisimple semihypergrups was studied by Corsini et al. [45]. Chvalina [25] started the concept of ordered semihypergroups. For more on ordered semihypergroups and ordered hyperstructures, one can refer [15], [16]. Walt [6] introduced the notions of prime and semiprime bi-ideals for an associative ring with unity. Roux [20] used the notions defined by Walt [6] and further studied the structure of a ring containing prime and semiprime bi-ideals. Due to these natural motivating facts, the results of this paper is a generalization of concepts inextricably connected with properties of some classical variety of hyperideals in semihypergroups that were also studied by Wallace [1], Hrmov'a [48], Roux [20], Kehayopulu [30], [31], [38], Saritha [47], Khan [40] and Changhphas [54] in semigroups, rings and other algebraic structures. For detailed elaborated work on ordered semigroups, hypersemigroups, ordered hypersemigroups, ordered $G$-semihypergroups, relative ideals and on their related concepts, one can refer the papers [2], [5], [7], [3], [8], [9], [10], [11], [4], [12], [13], [17], [27], [28], [29], [35], [39], [52], [53] and references of these papers.

\section{BASIC DEFINITIONS AND PREREQUISITES}

In this section, we introduce and recollect some basic definitions and results as indispensible and integrable prerequisites parts of this paper that will be needed as building blocks to construct and prove the results of this paper.

A hyperstructure $S$ is a nonempty set equipped with an hyperoperation

$$
\circ: S \times S \rightarrow P^{\star}(S) \mid(x, y) \rightarrow(x \circ y)
$$

on $S$ and an induced operation that of $S$ defined as follows:

$$
\star: P^{\star}(S) \times P^{\star}\left(S \rightarrow P^{\star}(S) \mid(X, Y) \rightarrow X \star Y\right.
$$

on $P^{\star}(S)$ such that

$$
X \star Y=\bigcup_{(x, y) \in X \times Y}(x \circ y)
$$

for any $X, Y \in P^{\star}(S)$. A hypergroupoid $S$ is denoted by $(S, \circ)$ since the operation " $\star$ " depends on the hyperoperation "॰". As an easy observation, we have

$$
X \subseteq Y \rightarrow X \star D \subseteq Y \star D, D \star X \subseteq D \star Y
$$

for any $X, Y, D \in P^{\star}(S)$ and $S \star S \subseteq S$. For a subset $X$ of a hypergroupoid $S$, we represent by $(X]$ the subset of $S$ defined as follows:

$$
(X]=\{s \in S: s \leq x \text { for some } \mathrm{x} \in \mathrm{X}\} .
$$


If " $\leq$ " is an order relation on a hypersemigroup $S$, we define the order relation " $\preceq "$ on $P^{\star}(S)$ as follows:

$$
\preceq:=\{(X, Y) \mid \forall x \in X \exists y \in Y \text { such that } \mathrm{x} \leq \mathrm{y}\} .
$$

Therefore, for $X, Y \in P^{\star}(S)$, we denote $X \preceq Y$ if for every $x \in X$, there exists $y \in Y$ such that $x \leq y$. This is clearly, reflexive and transitive relation on $P^{\star}(S)$.

A hyperstructure $(S, \circ)$ is called a semihypergroup if for all $x, y, z \in S,(x \circ y) \circ z=x \circ(y \circ z)$, i.e.,

$$
\bigcup_{m \in x \circ y} m \circ z=\bigcup_{n \in y \circ z} x \circ n .
$$

A nonempty subset $A$ of a semihypergroup $(S, \circ)$ is called a subsemihypergroup of $S$ if $A \star A \subseteq A$. A semihypergroup $(S, \circ)$ equipped with a partial order $" \leq$ " on $S$ that is compatible with semihypergroup operation " $\preceq$ " such that for all $x, y, z \in S$,

$$
x \leq y \Rightarrow z \circ x \preceq z \circ y \text { and } \mathrm{x} \circ \mathrm{z} \preceq \mathrm{y} \circ \mathrm{z},
$$

is called an ordered semihypergroup. Throughout this paper, $S$ will denote an semihypergroup(hypersemigroup) unless otherwise specified. Let $(S, \circ, \leq)$ be an ordered semihypergroup and let $X, Y$ be nonempty subsets of $S$, then we easily have the following:

(i): If $x \in X \star Y$, then $x \in x \circ y$ for some $x \in X, y \in Y$.

(ii): If $x \in X, y \in Y$, then $x \circ y \subseteq X \star Y$.

(iii): $X \subseteq(X]$.

(iv): If $X \subseteq Y$, then $(X] \subseteq(Y]$.

(v): $(X] \star(Y] \subseteq(X \star Y]$.

(vi): $(S]=((S]]$.

(vii): $((X] \star(Y]]=(X \star Y]$.

(viii): For every left (resp. right) G-hyperideal $I$ of $S,(I]_{G}=I$.

Now that if $I$ is a $G$-hyperideal of $S$, then the condition $I=(I]_{G}$ is equivalent to the condition: for $a \in I$ and $b \in G, b \leq a \Rightarrow b \in I$. Let $G \subseteq S$. The intersection of all left $G$-hyperideals of $S$ containing its nonempty subset $I$ is a $G$-hyperideal and is of the form: $L(I)=(I \cup G \star I]_{G}$. In a similar fashion, the intersection of all right $G$-hyperideals of $S$ containing its nonempty subset $I$ is a $G$-hyperideal and is of the form: $L(I)=(I \cup I \star G]_{G}$. For a $G$-bi-hyperideal $B$ of a partially ordered semihypergroup $(S, \circ, \leq)$, we note down the following notations:

$$
\begin{gathered}
L(B)=\{s \in B: S \star s \subseteq B\} \\
X(B)=\left\{s^{\prime} \in L(B): s^{\prime} \star S \subseteq L(B)\right\} .
\end{gathered}
$$

Definition 2.1. Suppose that $(S, \circ, \leq)$ is an ordered semihypergroup and $G \subseteq S$. Then, a nonempty subset $I$ of $S$ is called a right (resp. left) $G$-hyperideal of $S$ if

(i): $I \star G \subseteq I$ (resp. $G \star I \subseteq I)$;

(ii): if $x \in I$ and $G \ni y \leq x$, then $y \in I$, i.e., if $(I]_{G}=I$.

A subset of $S$ which is both a right and left $G$-hyperideal of $S$ is called a $G$-hyperideal of $S$. It is to be noted that $I \star G \subseteq I($ resp. $G \star I \subseteq I) \Longleftrightarrow x \circ g \subseteq I($ resp. $g \circ x \subseteq I)$ for every $x \in I$ and every $s \in S$. Obviously, every right(left) $G$-hyperideal of an ordered semihypergroup $S$ is a sub-semihypergroup of $S$. 
Definition 2.2. Let $S$ be an ordered semihypergroup and $G, T$ be any nonempty subsets of $S$. Then, $T$ is said to be a $G$-bi-hyperideal of $S$ if

(ii): $T \star G \star T \subseteq T$; and

(ii): for all $t \in T, G \ni g \leq t \Rightarrow g \in T$.

Definition 2.3. Let $S$ be an ordered hypersemigroup and $G \subseteq S$. Then, $S$ is called left $G$ regular(resp. right G-regular) if $g_{1} \leq g_{2} \circ g_{1}^{2}\left(\right.$ resp: $\left.g_{1} \leq g_{1}^{2} \circ g_{2}\right)$ for all $g_{1}, g_{2} \in G$. Equivalently:

(i): $g \in\left(G \star g^{2}\right]_{G}$ (resp., $\left.g \in\left(g^{2} \star G\right]_{G}\right)$ for all $g \in G$; and

(ii): $G \subseteq\left(G \star A^{2}\right]_{G}\left(r e s p . A \subseteq\left(A^{2} \star G\right]_{G}\right)$ for all $A \subseteq G$.

Definition 2.4. Suppose that $(S, \circ, \leq)$ is an ordered semihypergroup and $G \subseteq S$. Then, $S$ is called a $G$-idempotent semihypergroup if $G=(G \star G]_{G}$.

Definition 2.5. Suppose that $(S, \circ, \leq)$ is an ordered semihypergroup and $G \subseteq S$. Then, $S$ is called $G$-semisimple if for every $g \in G \exists a, b, c, d \in G$ such that

$$
\{g\} \preceq(a \circ g) \star(b \circ g) \star\{c\} \text { and } g \leq \mathrm{d} .
$$

Definition 2.6. Suppose that $(S, \circ, \leq)$ is an ordered hypersemigroup and $G \subset S$. Then, S called $G$-intra- regular if for every $g \in G$, there exist $a, b \in G$ such that $\{g\} \preceq(a \circ g) \star(g \circ b)$, that is, for every $g \in G$, there exists $a, b, c \in G$ such that $c \in(a \circ g) \star(g \circ b)$ and $g \leq c$.

\section{SOME PROPERTIES OF RELATIVE G-PRIME BI-HYPERIDEALS AND WEAKLY PRIME G-BI-HYPERIDEALS OF ORDERED HYPERSEMIGROUPS}

Theorem 3.1. Suppose that $P$ is a G-bi-hyperideal of a partially ordered semihypergroup $(S, \circ, \leq)$ and $G \subseteq S$. Then, $P$ is prime if and only if for any right $G$-hyperideal $R$ and for any left $G$-hyperideal $L$ of semihypergroup $S$,

$$
R \star L \subseteq P \Rightarrow R \subseteq P \text { or } \mathrm{L} \subseteq \mathrm{P} .
$$

Proof. Suppose that $P$ is a $G$-prime hyperideal of $S$. Further, suppose that $R$ and $L$ are a right $G$-hyperideal and a left $G$-hyperideal of $S$, respectively. Suppose that $R \nsubseteq P$. Then, there exists $s \in R \backslash P$. Let $s^{\prime} \in L$. Then, we have the following:

$$
s \star G \star s^{\prime} \subseteq R \star G \star L \subseteq R \star L \subseteq P .
$$

Since $s \notin P$, it implies by the given hypothesis that $s^{\prime} \in P$. Hence, $L \subseteq P$.

Conversely, suppose that for any right $G$-hyperideal $R$ and for any left $G$-hyperideal $L$ of $S$, we have the following:

$$
R \star L \subseteq P \Rightarrow R \subseteq P \text { or } \mathrm{L} \subseteq \mathrm{P} .
$$

Let $s, s^{\prime} \in G$ be such that $s \star G \star s^{\prime} \subseteq P$. By the following relation:

$$
(s \star G]_{G} \star\left(G \star s^{\prime}\right]_{G} \subseteq\left(s \star G^{2} \star s^{\prime}\right]_{G} \subseteq\left(s \star G \star s^{\prime}\right]_{G} \subseteq P,
$$

it implies by the given hypothesis that

$$
(s \star G]_{G} \subseteq P \text { or }\left(\mathrm{G} \star \mathrm{s}^{\prime}\right]_{\mathrm{G}} \subseteq \mathrm{P} .
$$

Let $(s \star G]_{G} \subseteq P$. Then, we have the following:

$$
s \circ s=s^{2} \subseteq P .
$$


Therefore,

$$
\begin{aligned}
R(s) \star L(s) & =(s \cup s \star G]_{G} \star(s \cup G \star s]_{G} \\
& \subseteq(s \circ s \cup s \star G \star s]_{G} \\
& \subseteq(s \circ s \cup s \star G]_{G} \\
& \subseteq P .
\end{aligned}
$$

So, $R(s) \subseteq P$ or $L(s) \subseteq P$. Hence, $s \in P$. We can prove the case for $\left(G \star s^{\prime}\right]_{G} \subseteq P$ in the similar fashion.

Theorem 3.2. Suppose that $P$ is a prime G-bi-hyperideal of a partially ordered semihypergroup $(S, \circ, \leq)$ and $G \subseteq S$. Then, $P$ is a left $G$-hyperideal of $S$ or $P$ is a right $G$-hyperideal of $S$.

Proof. Suppose that $P$ is a prime $G$-bi-hyperideal of a partially ordered semihypergroup $(S, \circ, \leq$ ). We can observe that $(G \star P]_{G}$ is a left $G$-hyperideal of $S$ and $(P \star G]_{G}$ is a right $G$-hyperideal of $S$. By the following relation:

$$
(P \star G]_{G} \star(G \star P]_{G} \star\left(P \star G^{2} \star P\right]_{G} \subseteq(P \star G \star P]_{G} \subseteq(P]_{G}=P .
$$

Therefore, by Theorem 3.1, we obtain the following:

$$
(G \star P]_{G} \subseteq P \text { or }(\mathrm{P} \star \mathrm{G}]_{\mathrm{G}} \subseteq \mathrm{P} .
$$

Hence, $G \star P \subseteq P$ or $\mathrm{P} \star \mathrm{G} \subseteq \mathrm{P}$.

Lemma 3.3. Suppose that $B$ is a $G$-bi-hyperideal of a partially ordered semihypergroup $(S, \circ, \leq)$ with zero 0 and $G \subseteq S$. Then, $L(B)$ is a left $G$-hyperideal of $S$.

Proof. We have $0 \in L(B)$, and $L(B) \neq \varnothing$. Suppose that $s \in L(B)$ and $s^{\prime} \in G$. We have

$$
s^{\prime} \circ s \subseteq G \star s \subseteq B
$$

Furthermore,

$$
G \star\left(s^{\prime} \circ s\right)=\left(G \star s^{\prime}\right) \star s \subseteq G \star s \subseteq B .
$$

Suppose that $s \in L(B)$ and $s^{\prime} \in G$ are such that $s^{\prime} \leq s$. Let $b \in G \star s^{\prime}$, then $b=s_{1} \circ s^{\prime}$ for some $s_{1} \in G$. By the following relation:

$$
b=s_{1} \circ s^{\prime} \preceq s_{1} \circ s \subseteq G \star s \subseteq B,
$$

we obtain $b \in B$. Hence, $s^{\prime} \in B$, and $L(B)$ is a left $G$-hyperideal of $S$.

Theorem 3.4. Suppose that $B$ is a G-bi-hyperideal of a partially ordered semihypergroup $(S, \circ, \leq)$ with zero 0 and $G \subseteq S$. Then, $X(B)$ is the unique largest $G$-hyperideal of $S$ contained in $B$.

Proof. We have $0 \in X(B)$. Suppose that $s, s^{\prime} \in G, b \in X(B)$. By Lemma 3.3, we have $s \circ b \subseteq L(B)$. As, $b \in X(B)$, we obtain $b \circ s^{\prime} \subseteq L(B)$. Therefore,

$$
(s \circ b) \circ s^{\prime}=s \circ\left(b \circ s^{\prime}\right) \subseteq L(B) .
$$


This proves that $G \star X(B) \subseteq X(B)$. By the following relation:

$$
b \circ s \subseteq L(B) \text { and }(\mathrm{b} \circ \mathrm{s}) \circ \mathrm{s}^{\prime} \subseteq \mathrm{b} \star \mathrm{G} \subseteq \mathrm{L}(\mathrm{B}) \text {, }
$$

we obtain

$$
X(B) \star G \subseteq X(B) .
$$

Suppose that $s \leq s^{\prime}$. As, $b \in L(B)$, we have $s \in L(B)$. Since,

$$
s \circ s^{\prime} \leq b \circ s^{\prime} \subseteq L(B), s \circ s^{\prime} \subseteq L(B) .
$$

Hence, $X(B)$ is a $G$-hyperideal of $S$. We observe that

$$
X(B) \subseteq L(B) \subseteq B .
$$

Lastly, suppose that $I$ is a $G$-hyperideal such that $I \subseteq B$. If $s \in A$, then $s \in B$ and $G \star s \subseteq$ $I \subseteq B$. Thus, $s \in L(B)$, and $I \subseteq L(B)$. Let $s \in A$. Therefore, $s \star G \subseteq I \subseteq L(B)$. Hence, $s \in X(B)$, and $I \subseteq X(B)$.

Theorem 3.5. Suppose that $P$ is a prime $G$ - bi-hyperideal of a partially ordered hypersemigroup $(S, \circ, \leq)$ with zero 0 and $G \subseteq S$. Then $X(P)$ is $G$-prime.

Proof. Suppose that $P$ is a prime $G$-bi-hyperideal of a partially ordered hypersemigroup $S$ such that $G \subseteq S$. Let $s, s^{\prime} \in G$ such that $I(s) \star I\left(s^{\prime}\right) \subseteq X(P)$. Then, by Theorem 3.1, we have $I(s) \subseteq X(P)$ or $I\left(s^{\prime}\right) \subseteq X(P)$. Hence, $s \in X(P)$ or $s^{\prime} \in X(P)$.

Theorem 3.6. Suppose that $B$ is a semiprime G-bi-hyperideal of a partially ordered semihypergroup $(S, \circ, \leq)$ and $G \subseteq S$. Then, we have the following:

(i): $L \star L \subseteq B \Rightarrow L \subseteq B$ for any left $G$-hyperideal $L$ of $S$.

(ii): $R \star R \subseteq B \Rightarrow R \subseteq B$ for any right $G$-hyperideal $R$ of $S$.

Proof. Suppose that $B$ is a semiprime $G$-bi-hyperideal of a partially ordered semihypergroup $(S, \circ, \leq)$. Let $L$ be a left $G$-hyperideal of $S$ such that $L \star L \subseteq B$. Let $s \in L$, then

$$
s \star B \star G \subseteq L \star G \star L \subseteq L \star L \subseteq B .
$$

By the given hypothesis, $s \in B$. In a similar fashion, we can prove the second condition.

Theorem 3.7. Suppose that $B$ is a semiprime G-bi-hyperideal of a partially ordered semihypergroup $(S, \circ, \leq)$ and $G \subseteq S$. Then, $X(B)$ is $G$-semiprime.

Proof. Suppose that $B$ is a semiprime $G$-bi-hyperideal of a partially ordered semihypergroup $(S, \circ, \leq)$ and $G \subseteq S$. Let $s \in G$ such that $I(s) \star I(s) \preceq X(B)$. Then, by Theorem 3.2, we have $I(s) \subseteq X(B)$. Hence, $s \in X(B)$.

Theorem 3.8. Suppose that $B$ is a semiprime G-bi-hyperideal of a partially ordered semihypergroup $(S, \circ, \leq)$ and $G \subseteq S$. Then, $B$ is a $G$-quasi-hyperideal of $S$.

Proof. Suppose that $B$ is a semiprime $G$-bi-hyperideal of a partially ordered semihypergroup $(S, \circ, \leq)$. It is sufficient to prove that

$$
B \star G \cap G \star B \subseteq G .
$$

Now, if $s \in B \star G \cap G \star B$, then

$$
s \star G \star s \subseteq(B \star G) \star G \star(G \star B) \subseteq B \star G \star B \subseteq B .
$$


Hence, $s \in B$, where $B$ is a $G$-quasi-hyperideal of $S$.

Theorem 3.9. Suppose that $(S, \circ, \leq)$ is a partially ordered semihypergroup and $G \subseteq S$. Then, $S$ is $G$-regular if and only if every $G$-bi-hyperideal of $S$ is $G$-semiprime.

Proof. Suppose that $S$ is $G$-regular. Let $B$ be a $G$-bi-hyperideal of $S$. Let $g \in G$ be such that $g \star G \star g \subseteq B$. Then, $(g \star G \star g]_{G} \subseteq(B]_{G}=B$. By the given hypothesis, $g \in(g \star G \star g]_{G} \subseteq B$. Hence, $B$ is $G$-semiprime.

Conversely, suppose that every $G$-bi-hyperideal of $S$ is $G$-semiprime. To prove that $S$ is $G$ regular, suppose that $g \in G$. We observe that $(g \star G \star g]_{G}$ is a $G$-bi-hyperideal of $S$. Therefore, by the given hypothesis, $(g \star G \star g]_{G}$ is $G$-semiprime. But $g \star G \star g \subseteq(g \star G \star g]_{G}$, then $g \in(g \star G \star g]_{G}$. Hence, $S$ is $G$-regular.

Theorem 3.10. Suppose that $(S, \circ, \leq)$ is an ordered hypersemigroup and $G \subseteq S$. Further, suppose that $X$ and $Y$ are $G$-hyperideals of $S$. Then, their intersection $X \cap Y$ is also a $G$ hyperideal of $S$.

Proof. Since $X$ is a right $G$-hyperideal and $Y$ a left $G$-hyperideal of $S$, we have $X \cap Y \neq \varnothing$. Consider, $x \in X$ and $y \in Y$. Then, $x \circ y \subseteq X \star G \subseteq X$ and $x \circ y \subseteq Y \star G \subseteq Y$, therefore, $x \circ y \subseteq X \cap Y$. Also, $x \circ y$ is a nonempty set, so the intersection $X \cap Y$ is a nonempty subset of $G$. Further, $(X \cap Y) \star G \subseteq X \star G \subseteq X$ and $(X \cap Y) \star G \subseteq Y \star G \subseteq Y$, therefore, $(X \cap Y) \star G \subseteq X \cap Y$. Let $a \in X \cap Y$ and $G \ni b \leq a$, then as $b \leq a \in X$, we obtain $b \in X$. Also, since $b \leq a \in Y$, we obtain $b \in Y$, thus $b \in X \cap Y$. Therefore, $X \cap Y$ is a right $G$-hyperideal of $S$. In a similar fashion, $X \cap Y$ is a left $G$-hyperideal of $S$. Hence, $X \cap Y$ is a $G$-hyperideal of $S$.

Theorem 3.11. Suppose that $(S, \circ, \leq)$ is an ordered hypersemigroup and $G \subseteq S$. Then, the $G$-hyperideals of $S$ are $G$-idempotent if and only if for any two $G$-hyperideals $X$ and $Y$ of $S$, we have $X \cap Y=(X \star Y]_{G}$.

Proof. Suppose that $X$ and $Y$ are $G$-idempotents of $S$. By Theorem 3.10, $X \cap Y$ is a $G$ hyperideal of $S$. By the given assumption, we obtain

$$
\begin{aligned}
X \cap Y & =((X \cap Y) \star(X \cap Y)]_{G} \\
& \subseteq(X \star Y]_{G} \\
& \subseteq(X \star G]_{G} \cap(G \star Y]_{G} \\
& \subseteq(X]_{G} \cap(Y]_{G}=X \cap Y
\end{aligned}
$$

Therefore, $X \cap Y=(X \star Y]_{G}$.

Conversely, suppose that $X$ is a $G$-hyperideal of $S$. By the given hypothesis, we obtain $X=$ $X \cap X=(X \star X]_{G}$. Hence, $X$ is $G$-idempotent

Theorem 3.12. Suppose that $(S, \circ, \leq)$ is an ordered semihypergroup and $G \subseteq S$. Let $X$ be a left $G$-hyperideal and $Y$ be a right $G$-hyperideal of $S$. Then, the set $(X \star Y]_{G}$ is a G-hyperideal of $S$. 
Proof. Since $X$ and $Y$ are nonempty subsets of $S, X \star Y$ and $(X \star Y]_{G}$ are nonempty subsets of $S$. Frthermore,

$$
\begin{aligned}
G \star(X \star Y]_{G} & =(G]_{G} \star(X \star Y]_{G} \\
& \subseteq(G \star(X \star Y)]_{G} \\
& =((G \star X) \star Y]_{G} \\
& \subseteq(X \star Y]_{G} .
\end{aligned}
$$

In a similar fashion,

$$
(X \star Y]_{G} \star G \subseteq(X \star Y]_{G} .
$$

Suppose that $a \in(X \star Y]_{G}$ and $G \ni b \leq a$. We obtain $a \leq m$ for some $m \in X \star Y$. As, $G \ni b \leq m \in X \star Y$, we obtain $b \in(X \star Y]_{G}$. Hence, $(X \star Y]_{G}$ is a $G$-hyperideal of $S$.

Corollary 3.13. Suppose that $S$ is an ordered semihypergroup and $G \subseteq S$. Further, suppose that $X, Y$ are $G$-hyperideals of $S$. Then, $(X \star Y]_{G}$ is a $G$-hyperideal of $S$.

Theorem 3.14. Suppose that $(S, \circ, \leq)$ is an ordered hypersemigroup and $G \subseteq S$. Then, the following are equivalent:

(i): $S$ is G-semiprime.

(ii): $g \in(G \star\{g\} \star G \star\{g\} \star G]_{G}$.

(iii): $P \subseteq(G \star P \star G \star P \star G]_{G}$ for every nonempty subset $P$ of $S$.

Proof. $(i) \Rightarrow(i i)$. Let $g \in G$. As, $S$ is $G$-semiprime, $\exists a, b, c, d \in G$ such that

$$
g \leq d \in\{a\} \star\{g\} \star\{b\} \star\{g\} \star\{d\} \subseteq G \star\{g\} \star G \star\{g\} \star G .
$$

Therefore, we obtain

$$
g \in(G \star\{g\} \star G \star\{g\} \star G]_{G} .
$$

$($ ii $) \Rightarrow\left(\right.$ iii). Suppose that $P \in P^{\star}(S)$ and $g \in P$. By (ii), we have

$$
g \in(G \star\{g\} \star G \star\{g\} \star G]_{G} \subseteq(G \star P \star G \star P \star G]_{G} .
$$

$($ iii $) \Rightarrow(i)$. Suppose that $g \in G$. By (iii), we have

$$
g \in\{g\} \subseteq(G \star\{g\} \star G \star\{g\} \star G]_{G} .
$$

Then, $g \leq d$ for some $d \in(G \star\{g\} \star G) \star\{g\} \star G, d \in m \circ n$ for some $m \in G \star\{g\} \star G, n \in\{g\} \star G$, $m \in s \circ b$ for some $s \in G \star\{g\}, b \in G, s \in a \circ g$ for some $a \in G$ and $n \in g \circ c$ for some $c \in G$. Therefore, we have

$$
\begin{aligned}
d \in m \circ n & =\{m\} \star\{n\} \\
& \subseteq(s \circ b) \circ\{n\} \\
& =\{s\} \star\{b\} \star\{n\} \\
& \subseteq(a \circ g) \star\{b\} \star(g \circ c),
\end{aligned}
$$

where $a, b, c, d \in G$ and $g \leq d$. Hence, $S$ is $G$-semisimple.

Theorem 3.15. Suppose that $(S, \circ, \leq)$ is an ordered hypersemigroup and $G \subseteq S$. Then, $S$ is $G$-semisimple if and only if the $G$-hyperideals of $S$ are $G$-idempotent. 
Proof. Suppose that $I$ is a $G$-hyperideal of $S$. Let $a \in I$ then, as $S$ is $G$-semisimple, we obtain

$$
a \in(G \star\{a\} \star G \star\{a\} \star G]_{G} .
$$

Then, $a \leq d$ for some

$$
d \in(G \star\{a\} \star G) \star(\{a\} \star G) .
$$

Then, $d \in p \circ q$ for some $p, q \in I$. As, $p, q \in I$ and $I$ is a sub-semihypergroup of $S$, we obtain

$$
p \circ q \subseteq I \star I \subseteq I .
$$

As, $a \leq d \in I$ and $I$ is a $G$-hyperideal of $S$, we have $a \in I$. Hence, the $G$-hyperideal of $S$ are $G$-idempotent.

Conversely, $g \in G$. By the given assumption, we have $I(g)=(I(g) \star I(g)]_{G}$. Therefore, we have

$$
\begin{aligned}
(I(g))^{2} & =\left((I(g))^{2}\right]_{G} \star I(g) \\
& =\left((I(g))^{2}\right]_{G} \star(I(g)]_{G} \\
& \subseteq\left((I(g))^{3}\right]_{G} .
\end{aligned}
$$

This further implies

$$
\begin{aligned}
(I(g))^{3} & =(I(g))^{2} \star I(g) \\
& \subseteq\left((I(g))^{3}\right]_{G} \star(I(g)]_{G} \\
& \subseteq\left((I(g))^{4}\right]_{G} .
\end{aligned}
$$

Moreover, this implies that

$$
(I(g))^{4} \subseteq\left((I(g))^{5}\right]_{G}
$$

Therefore, we have,

$$
\begin{aligned}
I(g) & =\left((I(g))^{2}\right]_{G} \\
& \subseteq\left(\left((I(g))^{3}\right]_{G}\right]_{G} \\
& =\left((I(g))^{3}\right]_{G} \\
& \subseteq\left(\left((I(g))^{4}\right]_{G}\right]_{G} \\
& =\left((I(g))^{4}\right]_{G} \\
& \subseteq\left(\left((I(g))^{5}\right]_{G}\right]_{G} \\
& =\left((I(g))^{5}\right]_{G} \\
& \subseteq(G \star I(g)]_{G} \subseteq(I(g)]_{G}=I(g) .
\end{aligned}
$$

This further implies that

$$
\begin{aligned}
(I(g))^{3} & =(g \cup G \star g \cup g \star G \cup G \star g \star G]_{G}^{3} \\
& \subseteq\left((g \cup G \star g \cup g \star G \cup G \star g \star G)^{2}\right]_{G} \star(g \cup G \star g \cup g \star G \cup G \star g \star G]_{G} \\
& \subseteq\left((G \star g \cup G \star g \star G]_{G} \star(g \cup G \star g \cup g \star G \cup G \star g \star G]_{G}\right. \\
& \subseteq((G \star g \cup G \star g \star G) \star(g \cup G \star g \cup g \star G \cup G \star g \star G)]_{G} \\
& \subseteq(G \star g \star G]_{G} .
\end{aligned}
$$


This implies that

$$
\begin{aligned}
(I(g))^{4} & \subseteq(G \star g \star G]_{G} \star(g \cup G \star g \cup g \star G \cup G \star g \star G]_{G} \\
& \subseteq\left(G \star g \star G \star g \cup G \star g \star G^{2} \star g \cup G \star g \star G \star g \star G \cup G \star g \star G^{2} \star g \star G\right]_{G} \\
& =(G \star g \star G \star g \cup G \star g \star G \star g \star G]_{G} .
\end{aligned}
$$

This implies that

$$
\begin{aligned}
(I(g))^{5} & \subseteq(G \star g \star G \star g \cup G \star g \star G \star g \star G]_{G} \star(g \cup G \star g \cup g \star G \cup G \star g \star G]_{G} \\
& \subseteq\left((G \star g \star G \star g \cup G \star g \star G \star g \star G]_{G} \star(g \cup G \star g \cup g \star G \cup G \star g \star G]_{G}\right. \\
& \subseteq(G \star g \star G \star g \star G]_{G} .
\end{aligned}
$$

Hence, we obtain

$$
\begin{aligned}
g \in I(g) & =\left((I(g))^{5}\right]_{G} \\
& \subseteq\left((G \star g \star G \star g \star G]_{G}\right]_{G} \\
& =(G \star g \star G \star g \star G]_{G} .
\end{aligned}
$$

Hence $S$ is $G$-semisimple.

Theorem 3.16. Suppose that $(S, \circ, \leq)$ is an ordered hypersemigroup and $G \subseteq S$. Then, the $G$-hyperideals of $S$ are $G$-weakly prime if and only if they are $G$-idempotents and form a chain.

Proof. Suppose that $I$ is a $G$-hyperideal of $S$. Then, $I=(I \star I]_{G}$. In fact, By Corollary 3.13, the set $(I \star I]_{G}$ is a $G$-hyperideal of $S$. As, $I \star I \subseteq(I \star I]_{G}$ and $(I \star I]_{G}$ is a $G$-weakly prime, we obtain

$$
I \subseteq(I \star I]_{G} \subseteq(I \star G]_{G} \subseteq(I]_{G}=I .
$$

Therefore, $I=(I \star I]_{G}$. Suppose that $X, Y$ are $G$-hyperideals of $S$. Then, $X \subseteq Y$ or $Y \subseteq X$. By Corollary 3.13, $(X \star Y]_{G}$ is a $G$-hyperideal of $S$. As, $\left(X \star Y \subseteq(X \star Y]_{G}\right.$ and $(X \star Y]_{G}$ is $G$-weakly prime, we obtain

$$
X \subseteq(X \star Y]_{G} \subseteq(G \star Y]_{G} \subseteq(Y]_{G}=Y .
$$

or

$$
Y \subseteq(X \star Y]_{G} \subseteq(X \star G]_{G} \subseteq(X]_{G}=X
$$

Conversely, suppose that $M$ is a $G$-hyperideal of $S$ and $X, Y$ are $G$-hyperideals of $S$ such that $X \star Y \subseteq M$. As the $G$-hyperideals of $\mathrm{S}$ are $G$-idempotent, by Theorem 3.11, we obtain

$$
X \cap Y=(X \star Y]_{G} .
$$

By the assumption, we have $X \subseteq Y$ or $Y \subseteq X$. If $X \subseteq Y$, then we have

$$
X=X \cap Y=(X \star Y]_{G} \subseteq(M]_{G}=M .
$$

If $Y \subseteq X$, then

$$
Y=X \cap Y=(X \star Y]_{G} \subseteq M
$$

Hence, $M$ is $G$-weakly prime.

Theorem 3.17. Suppose that $(S, \circ, \leq)$ is an ordered hypersemigroup and $G \subseteq S$. Then, for every nonempty subset $X$ of $S$, the set $(G \star X \star G]_{G}$ is a $G$-hyperideal of $S$. 
Proof. We know that $(G \star X \star G]_{G} \neq \varnothing$ and $(G \star X \star G]_{G} \subseteq S$. We have

$$
\begin{aligned}
(G \star X \star G]_{G} \star G & =(G \star X \star G]_{G} \star(G]_{G} \\
& \subseteq((G \star X \star G) \star G]_{G} \\
& =(G \star X \star(G \star G)]_{G} \\
& =(G \star X \star G]_{G} .
\end{aligned}
$$

In a similar fashion,

$$
G \star(G \star X \star G]_{G} \subseteq(G \star X \star G]_{G} .
$$

Also,

$$
\left((G \star X \star G]_{G}\right]_{G}=(G \star X \star G]_{G} .
$$

since $((M]]=(M]$ is true for any subset $M$ of $S$. Hence, $(G \star X \star G]_{G}$ is a $G$-hyperideal of $S$.

Theorem 3.18. Suppose that $(S, \circ, \leq)$ is an ordered hypersemigroup and $G \subseteq S$. Then, for any $G$-hyperideal of $S$, the following assertions are equivalent:

(i): I is G-weakly prime.

(ii): If $x, y \in G$, such that $(x \star G \star y]_{G} \subseteq I$, then either $x \in I$ or $y \in I$.

(iii): If $x, y \in G$ such that $I_{R}(x) \star I_{R}(y) \subseteq I$, then either $x \in I$ or $y \in I$.

(iv): if $X$ and $Y$ are left $G$-hyperideals of $S$ such that $X \star Y \subseteq I$, then either $X \subseteq I$ or $Y \subseteq I$.

(v): if $X$ and $Y$ are right $G$-hyperideals of $S$ such that $X \star Y \subseteq I$, then either $X \subseteq I$ or $Y \subseteq I$.

(vi): If $X$ is a right $G$-hyperideal and $Y$ is a left $G$-hyperideal of $S$ such that $X \star Y \subseteq I$, then either $X \subseteq I$ or $Y \subseteq I$.

Proof. $(i) \Rightarrow(i i)$. Suppose that $I$ is $G$-weakly prime. Let $x, y \in G$ such that $(x \star G \star y]_{G} \subseteq I$. Then, we have

$$
\begin{aligned}
(G \star x \star G]_{G} \star(G \star y \star G]_{G} & \subseteq\left(G \star x \star G^{2} \star y \star G\right]_{G} \\
& \subseteq(G \star(x \star G \star y) \star G]_{G} \\
& \subseteq\left(G \star(x \star G \star y]_{G} \star G\right]_{G} \\
& \subseteq(G \star I \star G]_{G} \\
& \subseteq(I]_{G}=I .
\end{aligned}
$$

Since $I$ is $G$-weakly prime, we have either $(G \star x \star G]_{G} \subseteq I$ or $(G \star y \star G]_{G} \subseteq I$. Let $(G \star x \star G]_{G} \subseteq I$. Then,

$$
\begin{aligned}
\left(I_{R}(x)\right)^{3} & =(x \cup G \star x \cup x \star G \cup G \star x \star G]_{G}^{3} \\
& \subseteq(x \cup G \star x \cup x \star G \cup G \star x \star G]_{G}^{2} \star(x \cup G \star x \cup x \star G \cup G \star x \star G]_{G} \\
& \subseteq(G \star x \cup G \circ x \star G]_{G} \star(x \cup G \star x \cup x \star G \cup G \star x \star G]_{G} \\
& \subseteq\left((G \circ x \cup G \circ x \circ G) \star(x \cup G \star x \cup x \star G \cup G \star x \star G]_{G}\right. \\
& \subseteq(G \star x \star G]_{G} \subseteq I .
\end{aligned}
$$


Therefore, we have

$$
\begin{aligned}
\left(\left(I_{R}(x)\right)^{2}\right]_{G} \star I_{R}(x) & =\left(\left((R(x))^{2}\right]_{G} \star\left(I_{R}(x)\right]_{G}\right. \\
& \subseteq\left(\left(I_{R}(x)\right)^{3}\right]_{G} \\
& \subseteq(I]_{G}=I .
\end{aligned}
$$

Since, $I$ is $G$-weakly prime and $\left(\left(I_{R}(x)\right)^{2}\right]_{G}$ is a $G$-hyperideal of $S$, either $\left(\left(I_{R}(x)\right)^{2}\right]_{G} \subseteq I$ or $I_{R}(x) \subseteq I$. Let $I_{R}(x) \subseteq I$. Then, $x \in I_{R}(x) \subseteq I$. Suppose that $\left(\left(I_{R}(x)\right)^{2}\right]_{G} \subseteq I$. Then, $\left(I_{R}(x)\right)^{2} \subseteq I$. Since, $I$ is $G$-semiprime, $I_{R}(x) \subseteq I$, and thus, $x \in I$. In a similar fashion, one can prove that if $(G \star y \star G]_{G} \subseteq I$, then $y \in I$.

$($ ii $) \Rightarrow($ iii $)$. Consider $x, y \in G$ such that $I_{R}(x) \star I_{R}(y) \subseteq I$. Then,

$$
\begin{aligned}
\left((x]_{G}\right) \star(G \star Y]_{G} & \subseteq\left((x \cup G \star x \cup x \star G \cup G \star x \star G]_{G}\right) \star\left((y \cup G \star y \cup y \star G \cup G \star y \star G]_{G}\right) \\
& \subseteq I .
\end{aligned}
$$

Therefore,

$$
(x \star G \star y]_{G} \subseteq\left(\left((x]_{G}\right) \star(G \star y]_{G}\right]_{G} \subseteq(I]_{G}=I .
$$

By (ii), we obtain either $x \in I$ or $y \in I$.

(iii) $\Rightarrow$ (iv). Suppose that $X, Y \subseteq G$ and $X, Y$ are right $G$-hyperideals of $S$ such that $X \star Y \subseteq I$ and $X \nsubseteq I$. Let $x \in X, x \notin I$ and $y \in Y$. Then,

$$
\begin{aligned}
I_{R}(x) & =(x \cup G \star x \cup x \star G \cup G \star x \star G]_{G} \\
& \subseteq(X \cup G \star X \cup X \star G \cup G \star X \star G]_{G} \\
& \subseteq(X \cup G \star X]_{G} .
\end{aligned}
$$

In a similar fashion,

$$
I_{R}(y) \subseteq(Y \cup G \star Y]_{G}
$$

Now,

$$
\begin{aligned}
I_{R}(x) \star I_{R}(y) & \subseteq\left((X \cup G \star X]_{G}\right) \star\left((Y \cup G \star Y]_{G}\right) \\
& \subseteq((X \cup G \star X) \star(Y \cup G \star Y)]_{G} \\
& =(X \star Y \cup X \star G \star Y \cup G \star X \star Y \cup G \star X \star G \star Y]_{G} \\
& \subseteq(X \star Y \cup G \star X \star Y]_{G} \\
& \subseteq(I \cup G \star I]_{G} \\
& \subseteq(I]_{G}=I .
\end{aligned}
$$

By (iii), either $I_{R}(x) \subseteq I$ or $I_{R}(y) \subseteq I$. This shows that $y \in I$. Hence, $Y \subseteq I$.

$($ iii $) \Rightarrow(v)$. The proof proceeds simply as above.

$($ iii $) \Rightarrow(v i)$. Consider a right $G$-hyperideal $X$ and a left $G$-hyperideal $Y$ of $S$ such that $X \star Y \subseteq$ $I$, and $X \nsubseteq I$. Again, consider $x \in X$ such that $x \notin I$. For $y \in Y$, since $I_{R}(x) \subseteq(X \cup G \star X]_{G}$ and

$$
I_{R}(y) \subseteq(Y \cup G \star Y \cup Y \star G \cup G \star Y \star G]_{G} \subseteq(Y \cup Y \star G]_{G},
$$


we have

$$
\begin{aligned}
I_{R}(x) \star I_{R}(y) & \subseteq((X \cup G \star X) \star(Y \cup Y \star G)]_{G} \\
& =(X \star Y \cup X \star Y \star G \cup G \star X \star Y \cup H \star X \star Y \star G]_{G} \\
& \subseteq(I \cup I \star G \cup G \star I \cup G \star I \star G]_{G} \\
& \subseteq(I]_{G}=I .
\end{aligned}
$$

By (iii), we have either $I_{R}(x) \subseteq I$ or $I_{R}(y) \subseteq I$. This shows that $y \in I$. Hence, $Y \subseteq I$. $(i v),(v)$ and $(v i) \Rightarrow(i)$ are straightforward.

\section{REFERENCES}

[1] A.D. Wallace, Relative ideals in semigroups I, Colloq. Math. 9 (1962) 55-61.

[2] A.D. Wallace, Relative ideals in semigroups. II: The relations of Green, Acta Math. Acad. Sci. Hungar. 14 (1963) 137-148. https://doi.org/10.1007/BF01901936.

[3] A. Basar, On some relative weakly hyperideals and relative prime bi-hyperideals in ordered hypersemigroups and in involution ordered hypersemigroups, Ann. Commun. Math. 3 (2020) 63-79.

[4] A. Basar, S.A. Khan, B. Satyanarayana, P.K. Sharma, A note on basic results on relative ordered $\Gamma$-ideals in ordered LA-Г-semigroups, GIS Sci. J. 9 (2022) 27-37.

[5] A. Basar, B. Satyanarayana, P.K. Sharma, Relative act hyperideals and relative hyperideals of hypersemigroups, J. Xi'an Univ. Arch. Technol. XIII (2021) 607-622.

[6] A.P.J. van der Walt, Prime and semiprime bi-ideals, Quaest. Math. 5 (1983) 341-345. https://doi.org/ 10.1080/16073606.1983.9632276.

[7] A. Basar, S. Ali, P.K. Sharma, B. Satyanarayana, M.Y. Abbasi, A study of ordered bi-Gamma-hyperideals in ordered Gamma-semihypergroups, Ikonion J. Math. 1 (2019) 34-45.

[8] A. Basar, Application of $(\mathrm{m}, \mathrm{n})-\Gamma$-hyperideals in characterization of LA- $\Gamma$-semihypergroups, Discuss. Math. - Gen. Algebra Appl. 39 (2019) 135. https://doi.org/10.7151/dmgaa.1304.

[9] A. Basar, M.Y. Abbasi, B. Satyanarayana, On generalized $\Gamma$-hyperideals in ordered $\Gamma$-semihypergroups, Fund. J. Math. Appl. 2 (2019) 18-23.

[10] A. Basar, S. Ali, M.Y. Abbasi, B. Satyanarayana and P. K. Sharma, On some hyperideals in ordered semihypergroups, J. New Theory, 29 (2019) 42-48.

[11] A. Basar, On some power joined $\Gamma$-semigroups, Int. J. Eng. Sci. Math. 8 (2019) 53-61.

[12] A. Basar, M.Y. Abbasi, On generalized bi- $\Gamma$-ideals in $\Gamma$-semigroups, Quasigroups Related Syst. 23 (2015) $181-186$.

[13] A. Basar and M. Y. Abbasi, On some properties of normal $\Gamma$-ideals in normal $\Gamma$-semigroups, TWMS J. Appl. Eng. Math. 9 (2019) 455-460.

[14] B. Davvaz, Semihypergroup Theory, Academic Press, 2016.

[15] D. Freni, Minimal order semihypergroups of type U on the right, II, J. Algebra, 340 (2011) 77-80.

[16] D. Heidari, B. Davvaz, On ordered hyperstructures, U.P.B. Sci. Bull. Ser. A, 73 (2011) 85-96.

[17] D.M. Lee, S.K. Lee, On Intra-regular ordered semigroups, Kangweon-Kyungki Math. J. 14 (2006) 95-100.

[18] E. Noether, Ideal theory in rings (Idealtheorie in ringbereichen), Translated by Daniel Berlyne, arXiv: 1401.2577v1 [math. RA], 2014.

[19] F. Marty, Sur uni generalization de la notion de groupe, 8th Congress Math. Scandinaves, Stockholm, (1934), 45-49.

[20] H.J. le Roux, A note on prime and semiprime bi-ideals of rings, Kyungpook Math. J. 35 (1995) 243-247.

[21] H.M. Edwards, The genesis of ideal theory, Arch. Hist. Ex. Sci. 23 (1980) 321-378.

[22] H.M. Edwards, Dedekind's invention of ideals, Bull. Lond. Math. Soc. 15 (1983) 8-17. https://doi.org/ 10.1112/blms/15.1.8.

[23] I. Kleiner, The genesis of the abstract ring concept, Amer. Math. Mon. 103 (1996) 417-424. https://doi. org/10.1080/00029890.1996.12004763. 
[24] I. Kleiner, Emmy noether and the advent of abstract algebra, In: Kleiner I. (eds) A History of Abstract Algebra, Birkhauser Boston, 2007.

[25] J. Chvalina, Commutative hypergroups in the sense of Marty and ordered sets, Proceedings of the Summer School on General Algebra and Ordered Sets 1994, 19-30.

[26] J. H. Barnett, Richard Dedekind and the Creation of an Ideal: Early Developments in Ring Theory, (2016), Abstract Algebra, 1.

[27] M.Y. Abbasi, A. Basar, Some properties of ordered 0-minimal (0, 2)-bi- $\Gamma$-ideals in po- $\Gamma$-semigroups, Hacet. J. Math. Stat. 44 (2015) 247-254. https://doi.org/10.15672/HJMS. 2015449100.

[28] M.Y. Abbasi, A. Basar, Weakly prime ideals in involution po-Г-semigroups, Kyungpook Math. J. 54 (2014) 629-638.

[29] M.Y. Abbasi, A. Basar, A note on ordered bi- $\Gamma$-ideals in intra-regular ordered $\Gamma$-semigroups, Afr. Mat. 27 (2016) 1403-1407. https://doi.org/10.1007/s13370-016-0419-y.

[30] N. Kehayopulu, On weakly prime ideals of ordered semigroups, Math. Japon. 35 (1990) 1051-1056.

[31] N. Kehayopulu, On prime, weakly prime ideals in ordered semigrous, Semigroup Forum, 44 (1992) 341-346. https://doi.org/10.1007/BF02574353.

[32] N. Kehayopulu, S. Lajos, M. Singelis, On intra-regular ordered semigroups, Pure Math. Appl. 4 (1993) 317-327.

[33] N. Kehayopulu, S. Lajos, G. Lepouras, A note on bi- and quasi-ideals of semigroups, ordered semigroups, Pure Math. Appl. 8 (1997) 75-81.

[34] N. Kehayopulu, On regular ordered semigroups, Math. Japon. 45 (1997) 549-553.

[35] N. Kehayopulu, On intra-regular ordered semigroups, Semigroup Forum, 46 (1993) 271-278.

[36] N. Kehayopulu and M. Tsingelis, On left regular ordered semigroups, Southeast Asian Bull. Math. 25 (2002) 609-615.

[37] N. Kehayopulu, On completely regular ordered semigroups, Sci. Math. 1 (1998) 27-32.

[38] N. Kehayopulu, On ordered hypersemigroups with idempotent ideals, prime or weakly prime ideals, Eur. J. Pure Appl. Math. 11 (2018) 10-22.

[39] N. Kehayopulu, On hypersemigroups, Pure Math. Appl. 25 (2015) 151-156.

[40] N.M. Khan, M.F. Ali, Relative bi-ideals and relative quasi ideals in ordered semigroups, Hacet. J. Math. Stat. 49 (2020) 950-961. https://doi.org/10.15672/hujms.624046.

[41] N.H. McCoy, Prime ideals of general rings, Amer. J. Math. 71 (1949) 823-833.

[42] O. Steinfeld, On ideal-quotients and prime ideals, Acta. Math. Acad. Sci. Hung. 4 (1953) 289-298.

[43] O. Steinfeld, Quasi ideals in rings and semigroups, vol. 10 of Disquisitiones Mathematicae Hungaricae, Akademiai Kiado, Budapest, Hungary, 1978.

[44] P. Corsini and V. Leoreanu, Applications of hyperstructure theory, Kluwer Academic Publishers, Dordrecht, Hardbound, 2003.

[45] P. Corsini, M. Shabir, T. Mahmood, Semisimple semihypergroups in terms of hyperideals and fuzzy hyperideals, Iran. J. Fuzzy Syst 8 (2011) 95-111

[46] P. Conrad, Ordered semigroups, Nagoya Math. J. 16 (1960), 51—64.

[47] R. Saritha, Prime and semiprime bi-ideals in ordered semigroups, Int. J.Algebra, 7 (2013) 839-845.

[48] R. Hrmova, Relative ideals in semigroups, Mat. Cas. 17 (1967) 206-223.

[49] R. A. Good and D. R. Hughes, Associated groups for a semigroup, Bull. Amer. Math. Soc. 58 (1952) 624-625.

[50] S. Lajos, Bi-ideals in semigroups I, A survey, PU. M. A. Ser. A2 (1991) 3-4.

[51] S. Lajos, Generalized ideals in semigroups, Acta Sci. Math. 2 (1961) 217-222.

[52] S. Kinugawa, J. Hashimoto, On relative maximal ideals in lattices, Proc. Japan Acad. 42 (1966) 1-4. https://doi.org/10.3792/pja/1195522165.

[53] T.K. Dutta, Relative ideals in groups, Kyungpook Math. J. 22 (1982) 1-5.

[54] T. Changhphas, On prime and semiprime bi-ideals in a partial ordered G-semigroup, Int. J. Pure. Appl. Math. 116 (2017) 717-725. 